\title{
Integral multidisciplinary rehabilitation treatment planning
}

\author{
Aleida Braaksma • Nikky Kortbeek · \\ Gerhard Post • Frans Nollet
}

Received: date / Accepted: date

\begin{abstract}
This paper presents a methodology to plan treatments for rehabilitation outpatients. These patients require a series of treatments by therapists from various disciplines. In current practice, when treatments are planned, a lack of coordination between the different disciplines, along with a failure to plan the entire treatment plan at once, often occurs. This situation jeopardizes both the quality of care and the logistical performance.

The multidisciplinary nature of the rehabilitation process complicates planning and control. An integral treatment planning methodology, based on an integer linear programming (ILP) formulation, ensures continuity of the rehabilitation process while simultaneously controlling seven performance indicators including access times, combination appointments, and therapist utilization. We apply our approach to the rehabilitation outpatient clinic of the Academic Medical Center (AMC) in Amsterdam, the Netherlands. Based on the results of this case, we are convinced that our approach can be valuable for decision-making support in resource capacity planning and control at many rehabilitation
\end{abstract}

A. Braaksma · N. Kortbeek

Stochastic Operations Research, University of Twente,

P.O. Box 217, 7500 AE Enschede, The Netherlands;

Center for Healthcare Operations Improvement and

Research (CHOIR), University of Twente;

Department of Quality Assurance and Process Innovation,

Academic Medical Center, P.O. Box 22660,

1100 DD Amsterdam, The Netherlands;

E-mail: \{a.braaksma,n.kortbeek\}@utwente.nl

G.F. Post

Discrete Mathematics and Mathematical Programming,

University of Twente

F. Nollet

Department of Rehabilitation, Academic Medical Center outpatient clinics. The developed model will be part of the new hospital information system of the AMC.

Keywords Rehabilitation treatment planning . Appointment scheduling · Patient flow · Integer linear programming

\section{Introduction}

Rehabilitation clinics treat patients recovering from injury, illness or disease. Patients require a series of treatments administered by therapists from various disciplines, such as physiotherapy, occupational therapy, social work, speech therapy, and psychology. According to the recent World Health Organization (WHO) report on disability [1], in high-income countries about $18 \%$ of the population lives with some form of disability, and the prevalence of disability is rising due to aging populations and the global increase in chronic health conditions. The expenditures for rehabilitation care have substantial pay offs including enhanced economic activity, health outcomes, educational achievements, and participation in community activities of people with disabilities [1]. Public spending on disability programs amounts to $1.2 \%$ of GDP for OECD countries and is particularly high in the Netherlands and Norway, where expenditures on disability account for approximately $5 \%$ of GDP [1]. The WHO [1] indicates improvement potential of rehabilitation care both in terms of quality and efficiency.

Because rehabilitation care is a multidisciplinary process, coordination within both the care process and the logistical organization is essential $[2,3]$. As in many health care processes, and rehabilitation in particular, planning deficiencies have a negative impact on both the quality of care and logistical efficiency [1,4]. The 
multidisciplinary nature of the rehabilitation process complicates planning and control. Naturally, the best quality of care is realized when the right treatments are provided at the right time [5]. Rehabilitation care professionals indicate that a short access time [6], a simultaneous start with the various disciplines, and the continuity of the rehabilitation process should be guaranteed. In addition, the complexity of rehabilitation care carries the risk of both undertreatment and overtreatment [7]. Despite the positive cost-effectiveness ratio of current rehabilitation care, both the WHO [1] and a recent improvement program for the Dutch rehabilitation sector [8] observe a large potential for rehabilitation care to be organized more efficiently and effectively. This paper connects with this improvement potential by presenting a planning methodology that enables the integral planning of multidisciplinary treatment plans. The effectiveness of this planning methodology is demonstrated by its application to a case study in the Academic Medical Center (AMC), a Dutch university hospital. Considerable enhancements in patientcenteredness, quality of care, and efficiency are achieved. By implementing the methodology, more patients can be treated with the same therapist capacity, and patients benefit from both a higher quality of care and a higher quality of service.

From the WHO report [1], we can conclude that the setting of the AMC rehabilitation clinic, and its organizational difficulties and logistical issues, are typical of rehabilitation care in general. In current AMC practice, several factors hinder the planning and control of rehabilitation care; of these factors, two main drivers are that planning is decentralized and that computerized support for the planning task is limited. All disciplines, or even therapists, manage their own agendas. Planners are supported by an electronic calendar system. However, the current state of this system comprises a database system that lacks the intelligence of a decision support system (see Section 3 for a more detailed discussion). Consequently, in many cases, a short access time and a so-called 'simultaneous start' cannot be realized. Moreover, the timely planning of followup appointments can be problematic, which can cause a discontinuity in the rehabilitation process. As a result, certain prescribed treatments may never be realized because they cannot be scheduled. In addition, outpatients have to visit the clinic more often than required, because appointments are spread out over several weekdays instead of combined into a single day. Concerning the system's logistical efficiency, planning deficiencies result in the suboptimal utilization of the valuable time of the therapists. We address these issues by developing a model for planning a series of appointments.

We identify three steps for improving a rehabilitation outpatient clinic's organization. The first step a clinic can take is to obtain insight into the demand and the supply of their rehabilitation care [1]. Although seemingly trivial, this insight is often lacking in practice. A clear perception of demand can be acquired by constructing treatment plans (per disease type or on an individual basis) [9], prescribing all treatments that should be realized during the course of a rehabilitation process. Insight in and control over supply can be gained via centrally managed therapist schedules [10]. As a second step, automated support of the planning task can yield further improvements $[1,11]$. A first requirement of a software tool is to enable planners to identify feasible planning proposals for individual patients based on their prescribed treatment plans [8]. Using such a decision support tool, the utilization of therapists could be made clear in an earlier stage, thereby enhancing the planning and control of this precious resource. In a third step, by exploiting operations research techniques, intelligent planning algorithms can be developed and implemented in the software tool to find planning proposals that are efficient for both patients and clinicians. Such tools also permit the evaluation of multiple planning strategies and provide a basis for rationalizing the required number of therapists, aligning therapist agendas, and determining the desired patient $\operatorname{mix}[12]$.

The present paper specifically addresses the third step noted above, as we present a method for planning series of appointments for rehabilitation outpatients based on an integer linear program (ILP). Using an ILP, multiple performance indicators are formulated for planning and are weighted according to a uniform strategy. To incorporate the particular characteristics and preferences of a certain organization, a planning methodology as developed in this paper needs to be context specific. Our basic approach is generically applicable to the rehabilitation sector, and the model can be customized for other multidisciplinary care facilities. As we have developed the planning methodology to support the rehabilitation outpatient clinic of the AMC, the ILP was developed in close cooperation with the rehabilitation care experts. The results of the AMC case demonstrate the application of such models for multidisciplinary treatment planning in the rehabilitation sector to be very promising.

This paper is organized as follows. Section 2 provides an overview of the related literature. Section 3 describes the case study setting. Section 4 presents the ILP model for planning a series of appointments. The 
planning methodology is applied to data from one of the treatment teams within the rehabilitation outpatient clinic of the AMC. We display the numerical results in Section 5 , followed by the discussion and conclusion in Section 6.

\section{Literature}

Appointment scheduling in health care is a topic that has received considerable attention in the literature. Two comprehensive surveys are provided in $[13,14]$. The literature has mostly focused on scheduling a given number of single appointments on a particular day for an individual service provider [13]. Gupta and Denton [14] identify several open challenges in appointment scheduling, prominent of which are planning coordinated packages of care for patients requiring treatment from several health services, scheduling in highly constrained situations, and incorporating patient preferences.

Rehabilitation planning has received little attention in the literature. To the best of our knowledge, there is no literature on scheduling series of appointments for rehabilitation outpatients in a multidisciplinary setting. Previous studies address an offline scheduling problem using a planning horizon of one day or one week and consider a single discipline [15-18]. We discuss these references in more detail. The scheduling challenge of sequencing a given set of physiotherapy treatments of multiple patients on a particular day is considered in [1517]. Chien et al. $[15,16]$ formulate this problem as a hybrid shop scheduling problem and solve it by developing a genetic algorithm [15], combined with data mining techniques in a later work [16]. Podgorelec and Kokol [17] present a scheduling algorithm based on genetic algorithms and machine learning. A time horizon of a week is considered in [18], in which one appointment per patient should be planned for a single discipline. Ogulata et al. [18] develop an integer linear programming (ILP) model that is broken down into three manageable hierarchical stages to resolve computational difficulty. In the first stage, patients are selected; in the second stage, patients are assigned to therapists; and in the third stage, patients are scheduled throughout a single day.

Methods have been developed for planning series of appointments for radiotherapy $[4,19]$ and chemotherapy [20] outpatients. For these patients, radiation treatments must be scheduled during a given number of weeks, strictly taking into account the required rest periods. Conforti et al. [4,19] present an ILP for radiotherapy treatment planning, minimizing access times while maximizing device utilization. Turkcan et al. [20] use a two stage ILP approach for solving a similar problem.
In the first stage, patients are assigned to days, and in the second stage, appointment times are given to all patients on their assigned days. The objectives are minimizing access times, treatment delays, and staff overtime. The main difference between radiotherapy treatment planning and rehabilitation treatment planning, is the single disciplinary nature of the former. In addition, the range of objectives involved in rehabilitation treatment planning is generally wider.

This paper presents a methodology for planning series of appointments for rehabilitation outpatients in a multidisciplinary setting, considering the numerous constraints and objectives that apply to rehabilitation treatment planning. The paper addresses the open challenges identified by Gupta and Denton [14] of planning coordinated packages of care, scheduling in highly constrained situations, and incorporating patient preferences.

\section{Background: the case study}

The rehabilitation outpatient clinic of the AMC employs 9 physicians and 30 therapists of various disciplines, who jointly perform approximately 10,000 consultations a year. Since 2008, the clinic has participated in an improvement program for the administration and planning practice by implementing a complete package of process redesign interventions, of which we will mention the main two. First, agenda management was centralized, and uniform schedules for the therapists were created. Second, standard treatment plans were formulated to standardize care processes, prevent undertreatment and overtreatment, and to obtain insight into demand. These two interventions are the starting point for the work presented in this paper, which introduces a planning methodology to enable optimal scheduling of the series of appointments prescribed in a treatment plan.

The patient flow, which is currently changing due to the planned introduction of treatment plans, is displayed in Figure 1. In the situation of 2008, the rehabilitation process started with a so-called intake consultation with a rehabilitation physician, who decided upon the disciplines that should be involved in the patient's care. The therapists determined the frequency and the timing of the treatments. After several weeks, the rehabilitation physician and the therapists discussed the condition of the patient during a multidisciplinary team (MDT) meeting. Together, they either decided to terminate or to continue the treatment.

As therapists strive to provide patients with the best possible care, the clinicians did report a risk of 


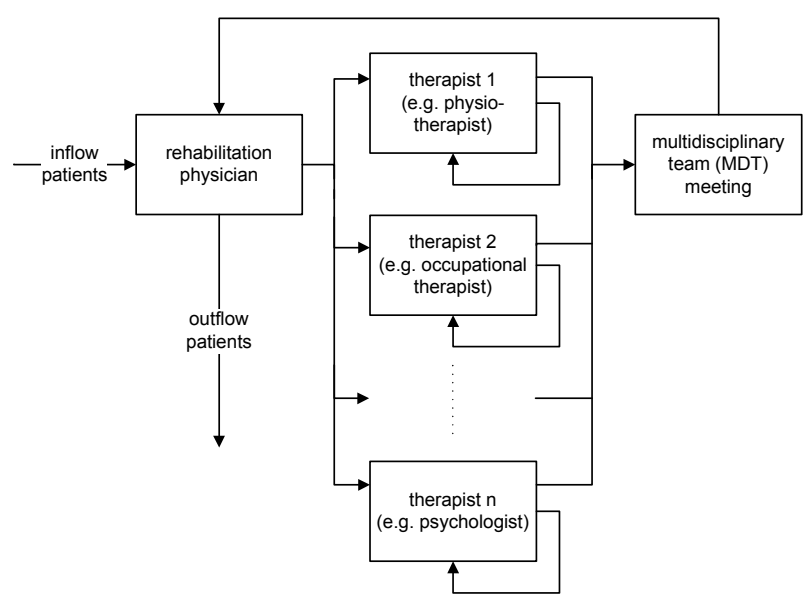

Fig. 1 Patient flow diagram

overtreatment. For each discipline, a follow-up appointment for the patient was only scheduled after the current treatment had taken place, resulting in scheduling on short notice. As this policy hampers the scheduling of an appointment at the prescribed moment, appointments were often scheduled later than prescribed, whereas the scheduling of certain appointments was omitted, thus resulting in undertreatment.

The introduction of treatment plans changes the patient flow. Following the intake consultation, the rehabilitation physician designs a treatment plan. The standard treatment plans form the basis for each patient treatment. In addition, physicians have the freedom to customize treatment plans if induced by individual patient needs. The treatment plan prescribes the disciplines that should be involved in the patient's treatment, the required number of treatments per discipline, the duration of each treatment and the week in which it should take place. Subsequently, all treatments up until the first MDT meeting are scheduled according to the treatment plan. During the MDT meeting, the rehabilitation physician and the therapists decide either to terminate the treatment of the patient or to design a plan for the continuation of the treatment. In the latter case, the required treatments are scheduled and the patient is scheduled to be discussed again during one of the upcoming MDT meetings.

Since January 2009, therapists and physicians of the rehabilitation outpatient clinic are grouped in three diagnosis-related treatment teams: Team Paediatrics, Team Neurology, and Team Orthopedics \& Traumatology. Each team has a dedicated planner who manages the schedules of all team members, so that treatment planning is centralized. Therapist schedules are standardized such that the time for patient care and the time for meetings or administration are synchro- nized among all therapists insofar as possible. Planners use the electronic calendar system X/Care (McKesson) to register appointments and select free appointment slots; therefore, planning is partially automated. However, X/Care has no flexible possibilities for planning treatment plans, let alone generating efficient planning proposals. When planning a treatment plan, planners have to consider the availability of therapists and of the patient in addition to patient preferences. Hence, whereas a single feasible planning proposal is already difficult to find, the planning task is further complicated by a complex set of constraints and preferences (see Section 4). Thus, finding a planning proposal for a complete treatment plan is a very time-consuming and cumbersome task. Planners indicate that they spend on average 15 minutes to find one feasible planning proposal for a multidisciplinary series of treatments for a patient. Therefore planning requests cannot be dealt with immediately. Instead planners tend to save up and execute planning requests once a week.

When the planner finds a feasible planning proposal, the appointments are fixed and the patient is informed via a letter. This process leaves very little room for patient preferences and is therefore not patient-centered. Moreover, if the patient is not available at some of the appointment times, the patient has to call the rehabilitation outpatient clinic and the planner has to reconsider the planning request. Some patients simply do not show up for their appointments without calling to cancel; it may be that such patients have not received the letter. The ability to execute a planning request promptly, when the patient is on the phone or at the desk, would leave more room to incorporate patient preferences, result in time savings for planners, and presumably reduce the number of no-shows.

In September and October 2009 we have performed baseline measurements of two performance indicators for all new patients starting their rehabilitation process (70 patients). As not all required information was available from the hospital databases, the rehabilitation planners manually registered the access time of each new patient and we assessed the case history of each individual patient. The average utilization of therapists during this period was $69 \%$, and the average utilization per discipline differed considerably (see Table 5). An access time within two weeks was achieved only for $22.9 \%$ of the patients. Of the 38 patients who required treatment with more than one discipline, $52.6 \%$ had a simultaneous start with the various disciplines. (For the exact definition of these performance indicators, see Section 4.1.)

Given the observations described, the current problems described in Section 1, and the results of the base- 
line measurements, it is to be expected that an intelligent planning methodology providing online decision support for the planners would be highly valuable to the rehabilitation outpatient clinic of the AMC.

\section{Methods}

In this section, the planning methodology is presented. First, the requirements of the model and the performance indicators are described, followed by the model formulation. The detailed mathematical formulation of the model is displayed in the appendix. Here, we discuss the framework of the model by describing the decision variables, the constraints, and the objective function. Figure 2 displays an overview of the model.

\subsection{Requirements of the model}

Given a patient with a prescribed treatment plan in addition to the skills and availabilities of the therapists, the model has to generate a planning proposal consisting of an assigned therapist and a start time for each appointment. The planning proposal, which must comply with the restrictions and preferences of the rehabilitation department, should result in a high-quality schedule for both the patient and the therapists involved.

In close cooperation with the clinicians of the rehabilitation outpatient clinic, we have formulated five performance indicators for the planning methodology, which are defined as follows:

- Access time. The number of days from the registration of a patient until the first appointment.

- Simultaneous start. The first appointments of a patient with the various disciplines take place within a pre-specified period (e.g., five working days).

- Lead time. The number of days from the first until the last appointment of a patient.

- Combination appointments. The number of days a patient has to visit the outpatient clinic compared to the minimal number of days necessary.

- Therapist utilization. The percentage of time available for patient care that is actually utilized for appointments.

In certain cases, a series of appointments can only be scheduled if some prescribed appointments are omitted. Because rejecting a planning request is far less desirable than omitting a small number of appointments, we allow for these appointments to not be scheduled if their number does not exceed a certain ratio per discipline (see Appendix). Moreover, clinicians indicate that quality of care cannot be guaranteed when the access time exceeds a certain threshold. To guarantee quality of care, a patient is referred to another clinic if the access time exceeds this threshold (see Appendix). Of course, it is highly preferable to reduce both of these occurrences to a minimum. Therefore, we also evaluate the performance of the following two indicators:

- Referred patients. The percentage of patients referred to another clinic.

- Unscheduled appointments. The percentage of appointments prescribed but not scheduled.

\subsection{Model formulation}

To obtain an optimization problem of manageable dimensions for which a provably optimal solution can be found within a reasonable time, we model the rehabilitation treatment planning problem as an integer linear program (ILP). In an ILP, restrictions specific to the rehabilitation treatment planning problem can be modeled appropriately, and multiple objectives can be weighted rationally.

The ILP is intended for scheduling a series of appointments for one patient at a time. Although this process may not produce the best overall schedules, it enables a direct response to a patient issuing a planning request, which is strongly preferred by the AMC for patient-centeredness reasons. For each series of appointments, the treatment plan prescribes the required number of treatments per discipline, the duration of each treatment, and the week in which it should take place. For each discipline, all appointments should be with the same therapist to ensure continuity of care. Scheduling a series of appointments exactly as prescribed by the treatment plan may not always be possible. Because rejecting a planning request is far less desirable than scheduling a series of appointments in a way that slightly deviates from the treatment plan, we allow for some scheduling flexibility. First, if an appointment cannot be scheduled in the week $(\mathrm{s})$ prescribed by the treatment plan, it may be scheduled a week earlier or later if these weeks do not already contain appointments with the same discipline. Second, as pointed out in Section 4.1, if the series can be scheduled except for a few appointments, we allow these appointments to not be scheduled if their number does not exceed a certain ratio per discipline. If a series cannot be scheduled despite this flexibility, we shift the planning horizon one week ahead and try again to schedule the series of appointments.

After each series of appointments, the patient is discussed during an MDT meeting, in which the decision is made either to terminate or to continue the treat- 
ment. In the latter case, another series of appointments needs to be scheduled after the MDT meeting. When scheduling the next series, information about the previous series may be relevant. This situation is described in detail in the appendix.

\subsubsection{Decision variables}

For each appointment within a series, we have to decide upon the assigned therapist and the starting time slot. We use the index $a$ for appointments, $h$ for therapists, and $t$ for time slots. The decision variables are as follows:

$x_{a h t}=\left\{\begin{array}{l}1 \text { if appointment } a \text { is assigned to therapist } h \\ \text { and starts in time slot } t \\ 0 \text { otherwise }\end{array}\right.$

\subsubsection{Constraints}

We distinguish several types of constraints:

Basic planning constraints. Appointments may not overlap, both the therapist and the patient have to be available for an appointment, and precedence relations between appointments must be satisfied.

Unscheduled appointments. For each discipline, a maximum of one in every $R$ appointments may be left unscheduled.

Therapist assignment. Per discipline, all appointments must be assigned to the same therapist. This socalled longitudinal continuity of care is a means of improving patient satisfaction and the outcomes of care [21].

Number of appointments per period. Multiple appointments with the same therapist may not be scheduled on the same day. Preferably, multiple appointments with one therapist are spread out evenly, both within and over weeks. The number of appointments with one therapist in a week is limited to $L$, and the number that may be scheduled on a single day is limited to $K$.

Start of the rehabilitation process. The access time of the patient should preferably be within $S$ weeks and may not exceed $C \cdot S$ weeks. To realize a simultaneous start, it is preferable that the first appointment with each discipline takes place within $V$ days of the patient's very first appointment.

Continuity of the rehabilitation process. An appointment should preferably be scheduled in the range of weeks prescribed by the treatment plan. However, it may be scheduled a week earlier or later if these weeks do not already contain appointments with the same discipline.
Patient preferences. Because combination appointments are high on the list of outpatient preferences [22], we strive to schedule the appointments on as few days as possible. The waiting time between appointments on the same day may not exceed $U$ time slots.

Recurring day and time. It is preferable that the appointments take place on the same day and time each week such that the patient has fewer days and times to remember.

Efficient filling of therapist schedules. We aim to schedule appointments right at the start or at the end of a session of the therapist, or right before or after an already scheduled appointment. This process prevents a break in the schedule between two consecutive appointments, that might be too short to fit in another appointment. Hence, we thereby minimize the number of referred patients and unscheduled appointments.

\subsubsection{Objective function}

The objective function consists of two main components. First, it contains the identified performance indicators (see Section 4.1). That is, we penalize the situations mentioned below. Each of these situations is characterized by one or more specific constraints in the appendix, referenced by the numbers in Figure 2 .

- the number of time slots by which the preferred access time is exceeded $(b \geq 0)$

- no simultaneous start realized with the various disciplines $(m=1$ in this case)

- the number of weeks by which the prescribed total duration of the series of appointments is exceeded $\left(z_{1}=1\right.$ in case of exceeding by two weeks or less, $z_{2}=1$ in case of exceeding between one and two weeks, $z_{3}=1$ in case of exceeding by more than two weeks)

- the number of extra days the patient has to visit the outpatient clinic because combination appointments have not been scheduled optimally $(p \geq 0)$

- the number of breaks created in the therapists' schedules ( $g_{a}=1$ if appointment $a$ causes a break)

- the number of unscheduled appointments $\left(n_{a}=1\right.$ if appointment $a$ is not scheduled)

The performance indicator referred patients is not contained in the objective function, because patients might only be referred when there are no feasible solutions. In addition to penalizing situations not adhering to the performance indicators, we penalize for three additional (undesirable) situations:

- the number of appointments that are scheduled a week earlier or later than prescribed in the treatment plan $\left(u_{a}=1\right.$ or $v_{a}=1$ in case appointment $a$ 


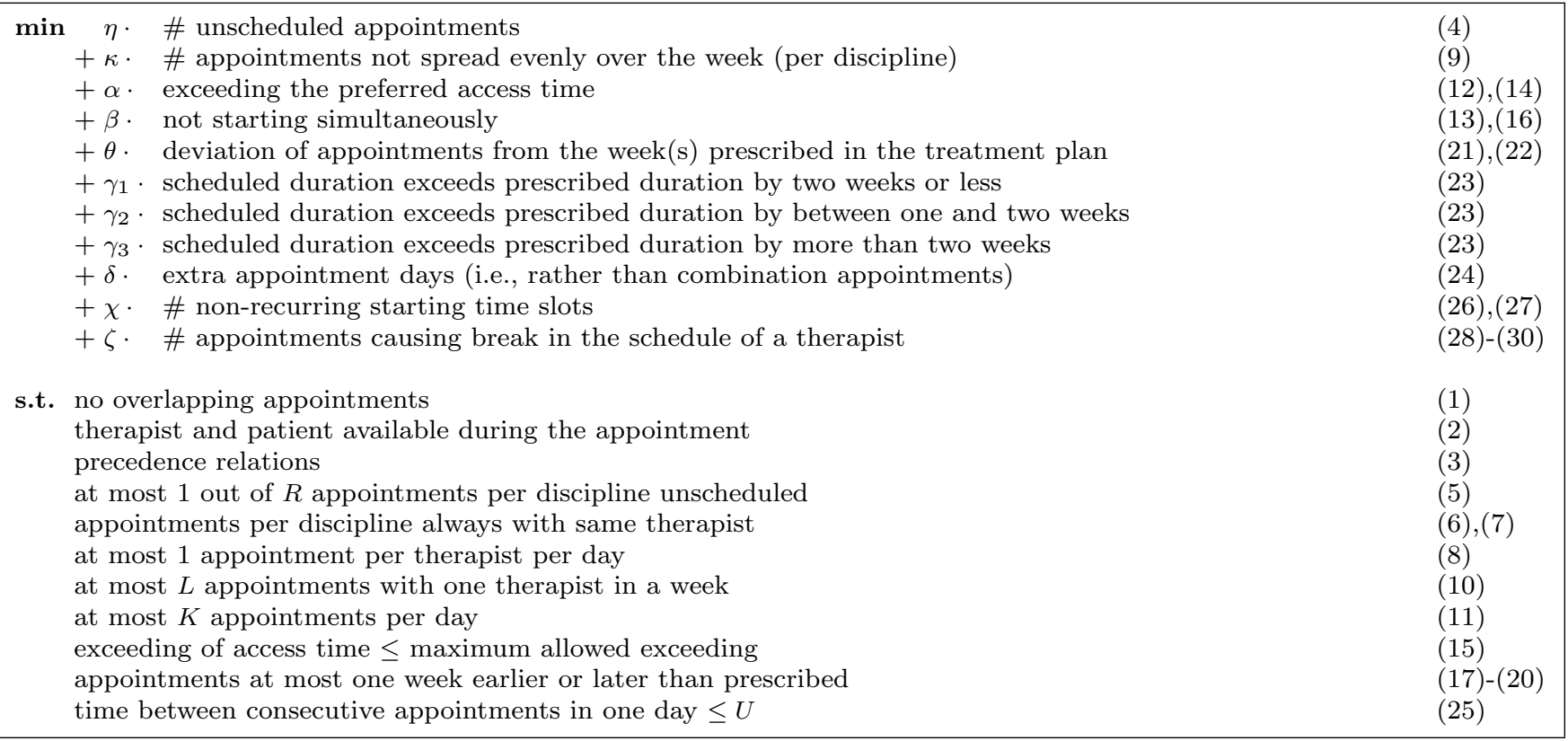

Fig. 2 Overview of the ILP (the numbers refer to the corresponding constraints in the appendix)

is scheduled a week earlier or later than prescribed, respectively)

- the number of appointments that take place one day after a previous appointment with the same therapist, such that the appointments per discipline are not spread out evenly over the week $\left(s_{a}=1\right.$ if this is the case for appointment $a$ )

- the number of unique (i.e. non-recurring) appointment times $(\mu \geq 0)$

The objective of the ILP is to minimize the sum of the weighted penalty costs, where $\eta, \ldots, \zeta$ are the weight factors and $D$ the number of time slots per day:

$$
\begin{aligned}
& \min \left\{\eta \cdot \sum_{a} n_{a}+\kappa \cdot \sum_{a} s_{a}+\alpha \cdot\left\lceil\frac{b}{D}\right\rceil+\beta \cdot m+\right. \\
& \left.\theta \cdot \sum_{a}\left\lceil\frac{u_{a}+v_{a}}{D}\right\rceil+\sum_{i=1}^{3} \gamma_{i} \cdot z_{i}+\delta \cdot p+\chi \cdot \mu+\zeta \cdot \sum_{a} g_{a}\right\} .
\end{aligned}
$$

One may observe that the objective function contains multiple goals that are possibly in conflict. For example, in some cases, it is possible to either schedule the first appointment within the preferred access time or to provide the patient a simultaneous start, but not both. As a second example, to optimally schedule combination appointments, it may be beneficial not to schedule certain appointments. By varying the weight factors, the relative importance of the various goals can be specified. The values of the weight factors can be set according to the preferences of the rehabilitation clinic in question. For each clinic, setting these values is part of configuring the ILP to the specific situation.

\section{Numerical results}

\subsection{Description of the test cases}

In this section, we apply the planning methodology to Team Neurology of the rehabilitation outpatient clinic in the AMC. Team Neurology mainly treats patients suffering from neuromuscular diseases, amyotrophic lateral sclerosis, post-polio syndrome, and cerebrovascular accidents.

After the intake consultation, the rehabilitation physician can assign the patient to a treatment plan in two ways. First, he can design an individual treatment plan for the patient. Second, he can assign the patient to one of the existing treatment plan blueprints. We test the methodology with seven treatment plan blueprints formulated by rehabilitation professionals. Table 1 shows the characteristics of these seven treatment plan blueprints. Each patient in our experiments is assigned to one of these seven blueprints. The relative frequency of the blueprints is based on hospital database information.

As Team Orthopedics \& Traumatology employs no psychologist, patients from Team Orthopedics \& Traumatology needing psychology are treated by the psychologist of Team Neurology. To represent the influence of care demands from these patients, we introduce a dummy treatment plan (see Table 1). As we do not incorporate the entire treatment plan of these patients because they are not assigned to Team Neurology, we 
Table 1 Characteristics of the treatment plan blueprints

$(\mathrm{PT}=$ physiotherapy, OT = occupational therapy, $\mathrm{ST}=$ speech therapy, $\mathrm{SW}=$ social work, $\mathrm{PS}=$ psychology $)$

\begin{tabular}{|c|c|c|c|c|c|c|c|c|c|}
\hline \multirow[t]{2}{*}{ Treatment plan } & \multirow[t]{2}{*}{ Patients } & \multirow[t]{2}{*}{ Series } & \multirow[t]{2}{*}{ Required for } & \multirow[t]{2}{*}{ Duration } & \multicolumn{5}{|c|}{ \# Appointments per discipline (\# hours) } \\
\hline & & & & & $\mathrm{PT}$ & OT & $\mathrm{ST}$ & SW & PS \\
\hline \multirow{3}{*}{$\begin{array}{l}\text { Amyotrophic lateral } \\
\text { sclerosis }\end{array}$} & $22 \%$ & 1 & $100 \%$ & 5 & $3(3.0)$ & $4(3.5)$ & $3(3.0)$ & $1(1.0)$ & \\
\hline & & 2 & $40 \%$ & 8 & $4(3.5)$ & $2(2.5)$ & $5(5.0)$ & $1(1.0)$ & \\
\hline & & 3 & $20 \%$ & 5 & $2(1.5)$ & $1(1.5)$ & $1(2.0)$ & $4(3.5)$ & \\
\hline \multirow[t]{3}{*}{ Post-polio syndrome } & $13 \%$ & 1 & $100 \%$ & 2 & $3(2.5)$ & $1(1.0)$ & & & \\
\hline & & 2 & $60 \%$ & 2 & $2(1.5)$ & $1(1.0)$ & & & \\
\hline & & 3 & $20 \%$ & 3 & $1(2.0)$ & $4(5.5)$ & & & \\
\hline \multirow{3}{*}{$\begin{array}{l}\text { Neuromuscular } \\
\text { diseases (other) }\end{array}$} & $4 \%$ & 1 & $100 \%$ & 4 & $4(4.0)$ & $1(1.5)$ & & $1(1.0)$ & \\
\hline & & 2 & $50 \%$ & 6 & $1(1.5)$ & $1(2.5)$ & & $2(1.5)$ & \\
\hline & & 3 & $20 \%$ & 10 & $2(1.5)$ & $3(3.0)$ & & $2(1.5)$ & \\
\hline \multirow{2}{*}{$\begin{array}{l}\text { Cerebrovascular } \\
\text { accidents }\end{array}$} & $17 \%$ & 1 & $100 \%$ & 3 & $3(3.0)$ & $2(3.0)$ & & $1(1.0)$ & $1(1.0)$ \\
\hline & & 2 & $50 \%$ & 7 & $4(2.0)$ & $2(3.0)$ & & $2(3.0)$ & $3(2.5)$ \\
\hline \multirow[t]{4}{*}{ Physiotherapy only } & $16 \%$ & 1 & $100 \%$ & 2 & $2(1.5)$ & & & & \\
\hline & & 2 & $70 \%$ & 2 & $1(0.5)$ & & & & \\
\hline & & 3 & $50 \%$ & 4 & $2(1.5)$ & & & & \\
\hline & & 4 & $30 \%$ & 5 & $2(1.5)$ & & & & \\
\hline \multirow{3}{*}{$\begin{array}{l}\text { Occupational therapy } \\
\text { only }\end{array}$} & $23 \%$ & 1 & $100 \%$ & 1 & & $1(1.0)$ & & & \\
\hline & & 2 & $50 \%$ & 4 & & $2(3.5)$ & & & \\
\hline & & 3 & $25 \%$ & 4 & & $2(3.0)$ & & & \\
\hline Ortho-trauma dummy & $5 \%$ & 1 & $100 \%$ & 4 & & & & & $4(4.0)$ \\
\hline
\end{tabular}

Explanation of the column items

Treatment plan:

Patients:

name of the treatment plan

Series:

percentage of patients assigned to this treatment plan

Required for:

number of the series of appointments within a treatment plan

after each series of appointments, during an MDT meeting the decision is made either to continue or to terminate the treatment of the patient; displayed is the percentage of patients continuing for the indicated series

Duration:

\# Appointments per discipline: prescribed duration in weeks of the series of appointments number of appointments within the series, for each discipline, including the total duration

exclude them from the summary scores on the various performance indicators.

Team Neurology employs nine therapists. Table 2 displays the availability of each therapist for direct outpatient care. Therapists spend their remaining time on indirect outpatient care (e.g., writing reports and ordering rehabilitation aids), meetings, inpatient care, and research. Because time for these activities is specifically reserved in their agendas, the sessions during which a therapist is indicated to be available for direct outpatient care are preferably completely filled with appointments. In Table 2, morning sessions last from 9:30 until 12:30 and afternoon sessions from 13:30 until 16:00. Therapists are not necessarily available for a full session. An indicator of therapist availability in Table 2 means that the therapist is available for at least one hour during that session. As therapists are not always available for outpatient care, certain (combination) appointments can only be made on specific days or at specific moments, which is quite restrictive for planning.
Table 3 lists the values used for the parameters in our experiments, which we set according to the restrictions and preferences of the AMC rehabilitation outpatient clinic. To be able to evaluate performance of the planning methodology from an organizational point of view, in our experiments we assume that patients are always available $\left(H_{t}=1 \quad \forall t\right)$. All appointments have a duration that is a multiple of 30 minutes. Therefore, in the experiments, each time slot has a length of 30 minutes.

Table 4 lists the values used for the weight factors in the experiments. To determine these values, the clinicians of the rehabilitation outpatient clinic scored the relative importance of each part of the objective function. As certain variables are binary whereas others are integer, we applied a normalization factor to each variable in order to generate comparable measures. These normalization factors, multiplied by their relative importance, produced the weight factor values listed in Table 4. 
Table 2 Weekly agenda for Team Neurology therapists

( $\mathrm{x}=$ therapist available for direct outpatient care)

$(\mathrm{PT}=$ physiotherapist, $\mathrm{OT}=$ occupational therapist, $\mathrm{ST}=$ speech therapist, $\mathrm{SW}=$ social worker, $\mathrm{PS}=$ psychologist $)$

\begin{tabular}{|c|c|c|c|c|c|c|c|c|c|c|c|}
\hline \multirow[t]{2}{*}{ Therapist } & \multicolumn{2}{|c|}{ Monday } & \multicolumn{2}{|c|}{ Tuesday } & \multicolumn{2}{|c|}{ Wednesday } & \multicolumn{2}{|c|}{ Thursday } & \multicolumn{2}{|c|}{ Friday } & \multirow[t]{2}{*}{ Total \# hours } \\
\hline & a.m. & p.m. & a.m. & p.m. & a.m. & p.m. & a.m. & p.m. & a.m. & p.m. & \\
\hline $\mathrm{PT} 1$ & $\mathrm{x}$ & & $\mathrm{x}$ & $\mathrm{x}$ & $\mathrm{x}$ & & $\mathrm{x}$ & $\mathrm{x}$ & $\mathrm{x}$ & & 18 \\
\hline PT 2 & $\mathrm{x}$ & & $\mathrm{x}$ & $\mathrm{x}$ & $\mathrm{x}$ & & $\mathrm{x}$ & $\mathrm{x}$ & & & 17 \\
\hline OT 1 & & $\mathrm{x}$ & & $\mathrm{x}$ & & $\mathrm{x}$ & $\mathrm{x}$ & & & $\mathrm{x}$ & 13 \\
\hline OT 2 & & & & & $\mathrm{x}$ & & & $\mathrm{x}$ & & & 6 \\
\hline OT 3 & $\mathrm{x}$ & $\mathrm{x}$ & $\mathrm{x}$ & & & & $\mathrm{x}$ & $\mathrm{x}$ & & & 13 \\
\hline OT 4 & & & & & $\mathrm{x}$ & $\mathrm{x}$ & & & $\mathrm{x}$ & $\mathrm{x}$ & 6 \\
\hline $\mathrm{ST}$ & $\mathrm{x}$ & $\mathrm{x}$ & & $\mathrm{x}$ & $\mathrm{x}$ & $\mathrm{x}$ & $\mathrm{x}$ & & & & 14 \\
\hline SW & $\mathrm{x}$ & $\mathrm{x}$ & $\mathrm{x}$ & $\mathrm{x}$ & & & $\mathrm{x}$ & $\mathrm{x}$ & & & 14 \\
\hline PS & $\mathrm{x}$ & $\mathrm{x}$ & & & & & $\mathrm{x}$ & $\mathrm{x}$ & & & 10 \\
\hline
\end{tabular}

\subsection{Experimental setup}

We use discrete-event simulation to evaluate the performance of our planning methodology. Prior to the actual simulation, we generate patient arrivals according to a Poisson process. The arrival rate of the Poisson process is set such that a desired therapist load is generated. For each patient, the release date and all treatment requirements are stored in a database. These requirements are generated based on the percentages listed in Table 1. Each patient is randomly assigned to one of the seven treatment plan blueprints. In addition, the required number of appointment series is drawn.

During the simulation, the patient with the earliest release date is selected from the database, and appointments are scheduled for this patient. Subsequently, the performance indicators are updated, the release date of the patient is set to the date of the MDT meeting in which the patient will be discussed, and the next patient is selected. As patients entering the system near the end of a simulation run cannot finish their treatment before the end of the run, we exclude the results of patients arriving during the last 20 weeks, which is the duration of the longest treatment plan.

We evaluate three scenarios. First, the base scenario, with an average therapist load of $70 \%$, is comparable to the therapist load during the baseline measurement observation period. To investigate the potential of the planning methodology to facilitate growth in demand, the average therapist load is set to $80 \%$ and $90 \%$ for the second and third scenarios, respectively. The average therapist utilization may differ slightly from the average therapist load due to three factors: first, the variation in the generation of patient arrivals; second, the percentage of unscheduled appointments; and third, the percentage of referred patients, with the latter two being preferably minimal.
Based on an analysis of the first five performance indicators (see Section 4.1) for five test runs, we set the warm-up period and the run length. The warmup period is determined by applying Welch's procedure [23] and is set to 2 years. This relatively long warm-up period results from the fact that the simulation starts from an empty system, whereas treatment plans have an average duration of 6.2 weeks, with the longest plan being 20 weeks. The run length (including the warm-up period) is set to 12 years. Based upon a desired half-width of $5 \%$ for the $95 \%$ confidence intervals of the performance indicators simultaneous start, lead time, combination appointments, and therapist utilization and a desired half-width of $10 \%$ for the $95 \%$ confidence interval of the performance indicator access time, the number of replications is set at 7 for Scenarios 1 and 2 and at 10 for Scenario 3.

The ILP was implemented in ILOG OPL 6.3 and solved using CPLEX 12.1. For our experiments we used a $2.27 \mathrm{GHz}$ Intel Core i3 ASUS Notebook with 4 GB RAM under a 64-bit version of Windows 7. Because the ILP is intended for scheduling a series of appointments for one patient at a time, numerous ILP instances must be solved during a simulation run. Most instances are solved to optimality within a few seconds. The average solving time is 14.2 seconds in Scenario 1 and decreases with increasing load, resulting in an average of 3.1 seconds for Scenario 3. In exceptional cases it can take several minutes to solve to optimality. This prolongation occurs in some of the cases in which a new multidisciplinary patient issues a planning request but therapist utilization is relatively low. Because the therapists to whom a new patient will be assigned have to be decided on and the therapist utilization is relatively low, the solution space is large in such cases.

To control the total duration of a simulation run, a CPU time limit of 600 seconds is applied to each ILP instance. Less than $0.005 \%$ of all instances are actually 
Table 3 Parameter values

\begin{tabular}{llr}
\hline Parameter & Description & Value \\
\hline$D$ & number of time slots per day & 13 \\
$R$ & number of appointments per discipline, of which at most one may be unscheduled & 5 \\
$L$ & maximum allowed number of appointments with one therapist in a week & 3 \\
$K$ & maximum allowed number of appointments on a single day & 3 \\
$S$ & number of weeks of preferred maximal access time & 2 \\
$W$ & number of time slots per week & 65 \\
$C$ & factor by which the exceeding of the access time is limited & 1 \\
$V$ & number of days within which all first appointments preferably take place (simultaneous start) & 5 \\
$T$ & number of time slots in the planning horizon & 325 \\
$U$ & maximum allowed waiting time between two consecutive (combination) appointments on a day & 1 \\
\hline
\end{tabular}

affected by this time limit. Hence, an optimal solution is identified in almost all cases, and for the remaining instances a near optimal solution is generated.

\subsection{Results}

Table 5 shows the experimental results for the three scenarios compared to the results of the baseline measurements. Clinicians are highly satisfied with the planning proposals generated by the model. The proposals generated are immediately implementable, without adjustment.

The planning methodology developed relates to the modified patient flow entailed by the introduction of the treatment plans (see Section 3). For the rehabilitation outpatient clinic, this new system differs so substantially from current practice, that there is no point in comparing the planning proposals generated by the model with the schedules that are currently being produced by the planners manually. Hence, the best we can do is to compare the results for the performance indicators realized by the model with the baseline measurements.

Note that the objective function of the ILP is the mechanism to direct the scheduling of appointments per individual patient. The value of the objective function

Table 4 Weight factor values

\begin{tabular}{llr}
\hline Weight factor & Objective & Value \\
\hline$\eta$ & unscheduled appointments & 500 \\
$\kappa$ & spreading of appointments & 1 \\
$\alpha$ & access time & 20 \\
$\beta$ & simultaneous start & 200 \\
$\theta$ & deviation from treatment plan & 1 \\
$\gamma_{1}$ & lead time & 50 \\
$\gamma_{2}$ & lead time & 150 \\
$\gamma_{3}$ & lead time & 300 \\
$\delta$ & combination appointments & 20 \\
$\chi$ & recurring day and time & 0 \\
$\zeta$ & therapist breaks & 5 \\
\hline
\end{tabular}

in itself is insignificant because we are interested in the realized planning product for the total patient population, which is evaluated by means of the formulated performance indicators. Results for the performance indicators simultaneous start and combination appointments only apply to patients being treated by multiple disciplines, and are therefore only reported for these patients. As seen in Table 1, 56\% of all patients follow a multidisciplinary treatment plan.

For four of the performance indicators, the results of the baseline measurements are not available for various reasons. During the baseline measurement observation period, the preferred duration of the rehabilitation process of a patient was not prescribed, such that we had no benchmark for the lead time. As appointments were scheduled one by one, it was hard to reconstruct which appointments could have been scheduled on the same day, complicating the measurement of the percentage of combination appointments. Because referred patients and unscheduled appointments were also not registered under the old system, these indicators were also unable to be measured during the baseline period.

The results of the baseline measurements and the experiments are displayed in Table 5 and Figures 3 and 4. With a therapist utilization comparable to the baseline measurements, the percentage of patients with an access time within two weeks increases from $22.9 \%$ to $98.9 \%$, representing an improvement of $76 \%$. The percentage of patients with a simultaneous start also improves from $52.6 \%$ to $100.0 \%$. Additionally, in nearly all cases (99.1\%), combination appointments are offered to patients. Although the results for lead time cannot be compared to the baseline measurements, based on the experiences of our clinicians we can state that the results of the experiments significantly outperform current practice; in addition, undertreatment is prevented. As strongly preferred, the percentages of referred patients and unscheduled appointments are very low.

When the therapist load is increased, the methodology still results in the production of a high-quality 
Table 5 Results of planning methodology compared to current practice

\begin{tabular}{|c|c|c|c|c|}
\hline Performance indicators & $\begin{array}{r}\text { Baseline } \\
\text { measurements }\end{array}$ & $\begin{array}{l}\text { Scenario } 1 \\
(\text { load } 70 \%)\end{array}$ & $\begin{array}{l}\text { Scenario } 2 \\
(\text { load } 80 \%)\end{array}$ & $\begin{array}{r}\text { Scenario } 3 \\
(\text { load } 90 \%)\end{array}$ \\
\hline $\begin{array}{l}\text { Access time } \\
\text { Percentage of patients with an access time } \leq 2 \text { weeks }\end{array}$ & $22.9 \%$ & $98.9 \%$ & $89.5 \%$ & $53.7 \%$ \\
\hline $\begin{array}{l}\text { Simultaneous start } \\
\text { Percentage of multidisciplinary patients having a } \\
\text { simultaneous start }\end{array}$ & $52.6 \%$ & $100.0 \%$ & $98.2 \%$ & $90.8 \%$ \\
\hline $\begin{array}{l}\text { Lead time } \\
\text { Percentage of patients with a lead time } \leq 10 \% \text { longer } \\
\text { than the prescribed duration }\end{array}$ & n.a. & $92.6 \%$ & $84.1 \%$ & $69.3 \%$ \\
\hline $\begin{array}{l}\text { Combination appointments } \\
\text { Percentage of combination appointments offered to } \\
\text { multidisciplinary patients }\end{array}$ & n.a. & $99.1 \%$ & $97.4 \%$ & $93.4 \%$ \\
\hline $\begin{array}{l}\text { Therapist utilization - overall } \\
\text { The percentage of time available for patient care } \\
\text { utilized for appointments }\end{array}$ & $69.3 \%$ & $70.1 \%$ & $79.3 \%$ & $87.4 \%$ \\
\hline $\begin{array}{lll}\text { Per discipline: } & \text { PT } & \text { physiotherapists } \\
& \text { OT } & \text { occupational therapists } \\
\text { ST } & \text { speech therapist } \\
\text { SW } & \text { social worker } \\
& \text { PS } & \text { psychologist }\end{array}$ & $\begin{array}{l}72.3 \% \\
72.1 \% \\
74.5 \% \\
60.7 \% \\
53.3 \%\end{array}$ & $\begin{array}{l}73.1 \% \\
73.0 \% \\
75.0 \% \\
61.6 \% \\
53.6 \%\end{array}$ & $\begin{array}{l}83.2 \% \\
83.0 \% \\
82.4 \% \\
69.7 \% \\
61.5 \%\end{array}$ & $\begin{array}{l}92.2 \% \\
91.1 \% \\
88.9 \% \\
77.5 \% \\
68.9 \%\end{array}$ \\
\hline $\begin{array}{l}\text { Referred patients } \\
\text { Percentage of patients referred to another clinic }\end{array}$ & n.a. & $0.00 \%$ & $0.29 \%$ & $2.47 \%$ \\
\hline $\begin{array}{l}\text { Unscheduled appointments } \\
\text { Percentage of appointments prescribed but not scheduled }\end{array}$ & n.a. & $0.12 \%$ & $0.25 \%$ & $0.33 \%$ \\
\hline
\end{tabular}

plan. With a therapist load of $80 \%$, simultaneous start and combination appointments have values above $95 \%$, and access time and lead time have values of $89.5 \%$ and $84.1 \%$, respectively. With a further increased therapist load of $90 \%$, simultaneous start and combination appointments continue to perform very well. However, access time, lead time and referred patients begin to deteriorate. To address this degradation in performance, we suggest three possible actions. First, a simple intervention to improve the continuity of care would be to discuss the patient during an MDT meeting in the week before the last scheduled appointments. In that way, the scheduling of follow-up appointments, if necessary, can take place a week earlier. Second, the values for weight factors in the objective function of the ILP might be adjusted, presumably at the cost of the other performance indicators. As pointed out earlier, in the end it is up to the health care professionals to decide upon the relative importance of the different performance indicators. Third, by reserving future capacity for patients already under treatment and requiring follow-up appointments, or for new patients, access time, lead time, and referred patients can possibly be improved. However, developing good reservation schemes is a study in itself, as the effects of reserving capacity on the various performance indicators are not trivial. Notably, with a therapist utilization of $87.4 \%$, the model in its current form signif- icantly outperforms the baseline measurements, which are realized at a therapist utilization of $69 \%$. Hence, by implementing the planning methodology, more patients can be treated with the same therapist capacity, and patients are offered both a higher quality of care and a higher quality of service.

\section{Discussion}

In this paper, we have presented a methodology for planning series of appointments for rehabilitation outpatients, that improves both the quality of care and logistical efficiency. These improvements in quality of care are realized through significantly shorter access times, an increased percentage of simultaneous starts, an enhanced continuity of care, a better coordination between disciplines via the introduction of treatment plans, and the elimination of both undertreatment and overtreatment. These findings are supported by the numerical results of a case study within the rehabilitation outpatient clinic of the AMC.

The planning methodology enhances patient-centeredness as it improves quality of care, provides patients with quick service, and yields a high percentage of combination appointments. Moreover, patient preferences, such as longitudinal continuity of care, are incorporated 


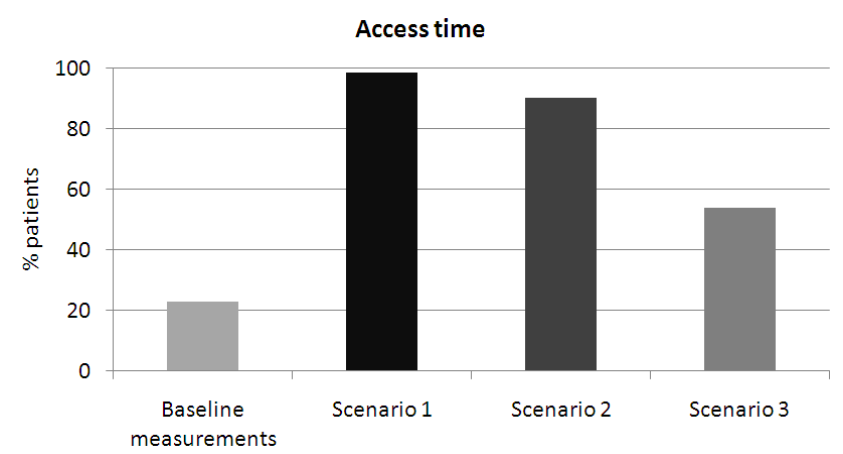

Fig. 3 Percentage of patients with an access time within 2 weeks

in the model. Multiple planning proposals can be generated quickly so that the patient is presented with a number of proposals to choose from. Different planning proposals can be generated by varying patient availability or by varying the weight factor values. Because a planning proposal can be generated within seconds, the model can deal with a planning request online, whereas, currently, planners tend to save up planning requests and execute the time-consuming and cumbersome planning task once a week. Dealing with a planning request on the fly reduces access times and provides prompt service to patients and up-to-date insight in terms of the demand for the rehabilitation clinic. This approach also presumably reduces the number of no-shows because patients are unquestionably notified of their appointments, and patients can immediately verify whether or not they are available at the proposed appointment times. Furthermore, the methodology induces cost savings as it reduces the time rehabilitation planners spend per planning request. Planners spend on average 15 minutes to put together one feasible planning proposal for a multidisciplinary series of treatments for a patient, whereas the model generates such a proposal within seconds.

Current health care planning systems do not support integral treatment planning. We have developed a prototype of a tool that does support such planning, and we have tested it in a rehabilitation outpatient clinic. Both patients and professionals are highly satisfied with the planning proposals generated by the model. This would not have been possible without formulating the model in cooperation with physicians, therapists, planners, and management of the rehabilitation outpatient clinic. Thus, despite the wide range of objectives and constraints, by carefully investigating these and formulating these in an ILP, our study has demonstrated that automated support of the planning task is possible. Based on the workability and the expected performance, the management of the AMC has decided

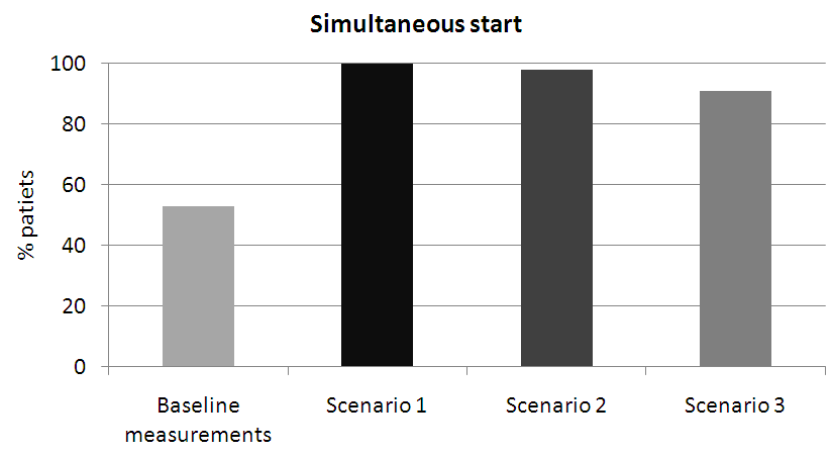

Fig. 4 Percentage of multidisciplinary patients with a simultaneous start

to include our planning methodology in the new hospital information system.

Planning multidisciplinary treatments is complex. The multidisciplinary character of rehabilitation care entails interaction between the agendas of the various therapists. The treatment of a patient with a particular discipline can only begin once the other disciplines required also have available capacity, and during the rehabilitation process appointments with the various disciplines have to be synchronized. As this interaction influences all performance indicators, aligning the capacities of the disciplines is of utmost importance. For the AMC case, the imbalance between the utilizations per discipline (see Table 5) may have a negative impact on the results, especially when therapist load is high, as an overloaded discipline blocks multidisciplinary patients from entering the clinic, whereas at the same time the other disciplines might have capacity available to accept those patients.

The AMC case is relatively small, with three disciplines (speech therapy, social work, and psychology) consisting of only one therapist. Although a larger case presumably results in a longer computation time, it increases planning flexibility, likely resulting in improved schedules. For example, there would be more freedom to select the therapists to whom the patient could be assigned, and as each discipline would presumably be present on most weekdays, there would be more possibilities for combination appointments. In addition, a clinic with a larger number of both therapists and patients would be less sensitive to demand fluctuations. Hence, we believe that, due to economies of scale, the potential of our approach for larger clinics is even greater than demonstrated in this paper.

Given the results of the AMC case, we are convinced that this methodology can be valuable to many rehabilitation outpatient clinics on the operational, tactical, and strategic planning levels. On the operational level, the ILP can be used for scheduling appointments. This 


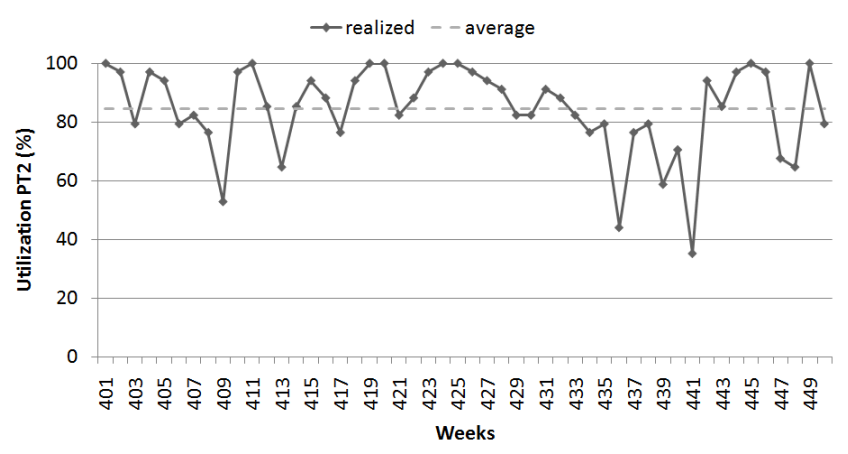

Fig. 5 Utilization of physiotherapist 2 during 50 weeks of a simulation run with a total length of 12 years (Scenario 3 )

process would require customization of the methodology to match the specific restrictions and preferences of each particular clinic. This customization is certainly possible as the ILP approach is suitable for changing or adding constraints and modifying the objective function. On the tactical level, by simulating the application of the methodology, therapist agendas can be aligned. The ILP method can also be beneficial on a strategic planning level, to rationalize the planning strategy and to expose the influence of increasing the relative importance of a particular performance indicator on overall performance. Moreover, the effects of changes in the case mix can be investigated, and insight can be acquired in rationally determining the relative capacities per discipline.

In future research, we will focus on three directions. First, as mentioned in Section 5.3, reserving capacity for both future patients and patients already under treatment might be a possibility to keep achieving excellent scores for all performance indicators under a high therapist load. Second, in our experiments we observed substantial variability in therapist utilization from week to week (see Figure 5). Balancing out of the utilization per therapist may be favorable. This balancing may possibly be achieved by taking the current utilization of therapists into account when assigning new patients to therapists. Third, as pointed out before, balancing the capacities of the various disciplines is of utmost importance. It may improve the performance of the system as a whole because it may positively affect all performance indicators. As aligning these capacities is not trivial due to the interactions between the disciplines, this area is an interesting direction for future research.

To conclude, this study has demonstrated that the world-wide organizational challenges recently established by the WHO can be well addressed by exploiting operations research techniques. Bringing together health care professionals and operations researchers can result in considerable improvements in both service quality and patient-centeredness for the rehabilitation sector.

\section{Acknowledgements}

1. The authors are grateful to the rehabilitation physicians and the therapists of the rehabilitation outpatient clinic of the Academic Medical Center Amsterdam (Kees Bijl in particular) who inspired us to take up this research topic, and we thank them for their involvement in the development and implementation of the methodology.

2. This research is supported by the Dutch Technology Foundation STW, applied science division of NWO and the Technology Program of the Ministry of Economic Affairs.

\section{Appendix}

This appendix contains the mathematical formulation of the ILP. Tables 6 and 7 provide a summary of the notation used. The presented formulation of the ILP is not entirely linear, but linearization is straightforward and is performed automatically by ILOG OPL, in which the ILP was implemented.

\section{Decision variables}

We use index $a$ for appointments, $h$ for therapists, and $t$ for time slots (see also Table 6). Each day is divided into $D$ time slots. Time slots are numbered consecutively, so $t=1$ is the first time slot on day one, $t=D+1$ is the first time slot on day two, and so on. We use the notation $\mathcal{T}_{d}$ for the set of time slots on day $d$ and $\mathcal{T}_{w}$ for the set of time slots in week $w$.

For each appointment within a series, we must select the therapist to whom the patient is assigned and the starting time slot. Hence, the decision variables are as follows:

$$
x_{a h t}=\left\{\begin{array}{l}
1 \text { if appointment } a \text { is assigned to therapist } h \\
\text { and starts in time slot } t, \\
0 \text { otherwise. }
\end{array}\right.
$$

To limit computation time, we do not construct decision variables $x_{a h t}$ that are not allowed. That is, $x_{a h t}$ is not constructed in the following cases:

- the disciplines of appointment $a$ and therapist $h$ do not match

- therapist $h$ is not available in time slot $t$

- the patient is not available in time slot $t$

- time slot $t$ is too near to the end of a day, such that appointment $a$ could not be finished before the end of the day if it were started in time slot $t$

- the patient is not treated by therapist $h$ (only applicable to patients who have already had treatments)

\section{Constraints}

In this section, we present the constraints of the model. Several types of constraints are considered. In addition to basic planning constraints, we distinguish constraints with respect to unscheduled appointments, therapist assignment, number of appointments per period, start and continuity of the rehabilitation process, patient preferences, recurring day and time, and the efficient filling of therapist schedules. 
Table 6 Indices and sets ILP

\begin{tabular}{llll}
\hline Index & Description & Set & Description \\
\hline$t, \hat{t}$ & time slots & $\mathcal{T}_{d}$ & time slots on day $d$ \\
$d$ & days & $\mathcal{T}_{w}$ & time slots in week $w$ \\
$w$ & weeks & $\mathcal{D}_{Y_{a}}$ & days in the week before \\
$h, \hat{h}$ & therapists & & week $Y_{a}$ \\
$c$ & disciplines & $\mathcal{D}_{Z_{a}}$ & days in the week after \\
$a, \hat{a}$ & appointments & & week $Z_{a}$ \\
\hline
\end{tabular}

\section{Basic planning constraints}

Let $M_{a}$ be the duration of appointment $a$. Any two appointments of the patient may not overlap. Starting with appointment $a$, other appointments $\hat{a}$ may not start at time slots in which appointment $a$ is taking place:

$\sum_{\hat{h}, \hat{a} \neq a} x_{\hat{a} \hat{h} \hat{t}}+x_{a h t} \leq 1, \quad \forall a, h, t, \hat{t} \mid t \leq \hat{t} \leq t+M_{a}-1$.

An appointment may only be scheduled if both the patient and the therapist are available. Let $G_{h t}$ be 1 if therapist $h$ is available in time slot $t$, and let $H_{t}$ be 1 if the patient is available in time slot $t$. Thus, we have to require the following:

$x_{a h t} \leq G_{h \hat{t}} \cdot H_{\hat{t}}, \quad \forall a, h, t, \hat{t} \mid t \leq \hat{t} \leq t+M_{a}-1$.

The treatment plan may contain precedence relations between certain appointments. Let parameter $B_{a \hat{a}}$ be 1 if appointment $a$ should take place before $\hat{a}$ and 0 otherwise. To satisfy the precedence relations, we have to require the following:

$\sum_{\hat{t} \leq t} B_{a \hat{a}} \cdot x_{\hat{a} \hat{h} \hat{t}} \leq 1-x_{a h t}, \quad \forall a, \hat{a}, h, \hat{h}, t$.

\section{Unscheduled appointments}

As pointed out in Section 4.2, we allow a limited number of unscheduled appointments. The variable $n_{a}$ is 1 if appointment $a$ is not scheduled and 0 otherwise:

$\sum_{h, t} x_{a h t}=1-n_{a}, \quad \forall a$.

As it is undesirable to omit appointments, the number of unscheduled appointments is penalized in the objective function. For each discipline $c$, the number of unscheduled appointments is limited to a maximum of 1 in every $R$ appointments that are prescribed in the treatment plan. Recall that when scheduling a series of appointments for a patient, previous series of appointments may already have been scheduled for this patient in the past. Let $P_{c}$ be the number of appointments prescribed for discipline $c$ in previous series, $Q_{c}$ the number of those appointments that have not been scheduled, and $O_{c}$ the number of appointments prescribed in the current series. Furthermore, $I_{a c}$ is 1 if appointment $a$ belongs to discipline $c$ and 0 otherwise. Thus, for the limitation on the number of unscheduled appointments per discipline, we have the following:

$Q_{c}+\sum_{a} I_{a c} \cdot n_{a} \leq \frac{1}{R}\left(P_{c}+O_{c}\right), \quad \forall c$.

\section{Therapist assignment}

For each discipline, all appointments have to be assigned to the same therapist. This so-called longitudinal continuity of care is a means of improving patient satisfaction and outcomes of care [21]. We introduce the auxiliary variables $y_{h}$ that equal 1 if the patient is assigned to therapist $h$ and 0 otherwise:

$x_{a h t} \leq y_{h}, \quad \forall a, h, t$.

Let parameter $J_{h c}$ be 1 if therapist $h$ belongs to discipline $c$ We enforce longitudinal continuity of care by the following equation:

$\sum_{h} J_{h c} \cdot y_{h} \leq 1, \quad \forall c$.

By not constructing decision variables $x_{a \hat{h} t}$ for therapists $\hat{h}$ who do not treat the patient, we will require that $y_{h}=1$ if the patient has had treatments from therapist $h$ in previous series.

\section{Number of appointments per period}

Multiple appointments with the same therapist may not be scheduled on the same day $d$. Let $A_{h t}$ be 1 if an appointment of the previously scheduled series of the patient is assigned to therapist $h$ and starts in time slot $t$. Recall that $\mathcal{T}_{d}$ denotes the set of time slots on day $d$. Then, we require the following:

$\sum_{t \in \mathcal{T}_{d}}\left(A_{h t}+\sum_{a} x_{a h t}\right) \leq 1, \quad \forall h, d$

Preferably, multiple appointments with one therapist are evenly spread over a week. Hence, we will penalize situations in which appointments with one therapist are scheduled on consecutive days. Let $s_{a}$ be 1 if appointment $a$ is scheduled such that it takes place one day after a previous appointment with the same therapist. We penalize $s_{a}$ in the objective function. Let $d_{1}$ denote the day after the day of time slot $t$. Therefore, the constraint is as follows:

$\sum_{\hat{t} \in \mathcal{T}_{d_{1}}}\left(A_{h \hat{t}}+\sum_{\hat{a}} x_{\hat{a} h \hat{t}}\right)+x_{a h t} \leq 1+s_{a}, \quad \forall a, h, t$.

To also enhance the spreading out of the treatments per discipline over weeks, the number of appointments with one therapist in a week is limited to $L$. Remember that $\mathcal{T}_{w}$ denotes the set of time slots in week $w$. Hence, the constraint is as follows:

$\sum_{t \in \mathcal{T}_{w}}\left(A_{h t}+\sum_{a} x_{a h t}\right) \leq L, \quad \forall h, w$.

As treatments may be strenuous for the patient, the number of appointments that may be scheduled on a single day is limited to $K$. We introduce auxiliary variables $e_{d}$ which are 1 if one or more appointmentsare scheduled on day $d$ and 0 otherwise:

$\sum_{t \in \mathcal{T}_{d}} \sum_{h}\left(A_{h t}+\sum_{a} x_{a h t}\right) \leq K \cdot e_{d}, \quad \forall d$. 
Table 7 Parameters and variables ILP

\begin{tabular}{|c|c|c|c|}
\hline Parameters & Description & Variables & Description \\
\hline \multicolumn{2}{|c|}{ Binary parameters } & \multicolumn{2}{|c|}{ Binary variables } \\
\hline$G_{h t}$ & 1 if therapist $h$ is available in time slot $t$ & \multirow[t]{2}{*}{$x_{a h t}$} & \multirow{2}{*}{$\begin{array}{l}1 \text { if appointment } a \text { is assigned to therapist } h \text { and } \\
\text { starts in time slot } t\end{array}$} \\
\hline$H_{t}$ & 1 if the patient is available in time slot $t$ & & \\
\hline$B_{a \hat{a}}$ & 1 if appointment $a$ should take place before $\hat{a}$ & $n_{a}$ & 1 if appointment $a$ is not scheduled \\
\hline$I_{a c}$ & 1 if appointment $a$ belongs to discipline $c$ & $y_{h}$ & 1 if the patient is assigned to therapist $h$ \\
\hline$J_{h c}$ & 1 if therapist $h$ belongs to discipline $c$ & $s_{a}$ & \\
\hline$A_{h t}$ & $\begin{array}{l}1 \text { if an appointment of the previously scheduled } \\
\text { series of the patient is assigned to therapist } h \\
\text { and starts in time slot } t\end{array}$ & $e_{d}$ & $\begin{array}{l}\text { previous appointment with the same therapist } \\
1 \text { if appointments for the patient are scheduled on } \\
\text { day } d\end{array}$ \\
\hline$F_{a c}$ & $\begin{array}{l}1 \text { if appointment } a \text { is the first appointment for } \\
\text { discipline } c \text { according to the treatment plan }\end{array}$ & $m$ & $\begin{array}{l}1 \text { if the patient has no simultaneous start with } \\
\text { the various disciplines }\end{array}$ \\
\hline$N$ & 1 if the patient is a new patient & \multirow[t]{2}{*}{$q_{a}$} & \multirow{2}{*}{$\begin{array}{l}1 \text { if appointment } a \text { may not be scheduled a week } \\
\text { earlier than prescribed in the treatment plan }\end{array}$} \\
\hline$E_{t \hat{t}}$ & 1 if time slot $t$ and $\hat{t}$ are on the same day & & \\
\hline \multicolumn{2}{|c|}{ General integer parameters } & $r_{a}$ & $\begin{array}{l}1 \text { if appointment } a \text { may not be scheduled a week } \\
\text { later than prescribed in the treatment plan }\end{array}$ \\
\hline$D$ & number of time slots per day & \multirow[t]{2}{*}{$z_{1}$} & \multirow{2}{*}{$\begin{array}{l}1 \text { if prescribed duration of the series of } \\
\text { appointments is exceeded by two weeks or less }\end{array}$} \\
\hline$M_{a}$ & duration of appointment $a$ & & \\
\hline$R$ & $\begin{array}{l}\text { number of appointments per discipline, of } \\
\text { which at most one may be unscheduled }\end{array}$ & $z_{2}$ & $\begin{array}{l}1 \text { if exceeding of prescribed duration of series of } \\
\text { appointments is between one and two weeks }\end{array}$ \\
\hline$P_{c}$ & $\begin{array}{l}\text { number of appointments prescribed for } \\
\text { discipline } c \text { in previous series }\end{array}$ & $z_{3}$ & $\begin{array}{l}1 \text { if prescribed duration of series of appointments } \\
\text { is exceeded by more than two weeks }\end{array}$ \\
\hline \multirow[t]{2}{*}{$Q_{c}$} & number of appointments prescribed but not & $\tau_{t}$ & \multirow{2}{*}{$\begin{array}{l}1 \text { if appointment } a \text { causes idle time in the } \\
\text { schedule of the therapist beforehand }\end{array}$} \\
\hline & $\begin{array}{l}\text { scheduled for discipline } c \text { in previous series } \\
\text { number of appointments prescribed for }\end{array}$ & $i_{a}$ & \\
\hline$O_{c}$ & discipline $c$ in the current series & \multirow{2}{*}{$j_{a}$} & \multirow{2}{*}{$\begin{array}{l}1 \text { if appointment } a \text { causes idle time in the } \\
\text { schedule of the therapist afterwards }\end{array}$} \\
\hline \multirow[t]{2}{*}{$L$} & maximum allowed number of appointments & & \\
\hline & with one therapist in a week & \multirow[t]{3}{*}{$g_{a}$} & \multirow{3}{*}{$\begin{array}{l}1 \text { if appointment } a \text { causes idle time in the } \\
\text { schedule of the therapist both beforehand and } \\
\text { afterwards }\end{array}$} \\
\hline$K$ & $\begin{array}{l}\text { maximum allowed number of appointments } \\
\text { on a single day }\end{array}$ & & \\
\hline$S$ & preferred maximal access time (\# weeks) & & \\
\hline$W$ & number of time slots per week & \multicolumn{2}{|c|}{ General integer variables } \\
\hline$C$ & $\begin{array}{l}\text { factor by which the exceeding of the access } \\
\text { time is limited }\end{array}$ & $f$ & $\begin{array}{l}\text { number of the starting time slot of the first } \\
\text { appointment }\end{array}$ \\
\hline$V$ & $\begin{array}{l}\text { number of days within which all first appoint- } \\
\text { ments preferably take place (simultaneous start) }\end{array}$ & $k$ & $\begin{array}{l}\text { number of the day on which the first } \\
\text { appointment is scheduled }\end{array}$ \\
\hline$Y_{a}$ & $\begin{array}{l}\text { number of the first week in which appointment } \\
a \text { may be scheduled }\end{array}$ & $b$ & $\begin{array}{l}\text { number of time slots by which the preferred } \\
\text { access time is exceeded }\end{array}$ \\
\hline$Z_{a}$ & $\begin{array}{l}\text { number of the final week in which appointment } \\
a \text { may be scheduled }\end{array}$ & $u_{a}$ & $\begin{array}{l}\text { number of time slots that appointment } a \text { is } \\
\text { scheduled before week } Y_{a}\end{array}$ \\
\hline$\Phi$ & $\begin{array}{l}\text { number of days that have passed since the } \\
\text { start of the treatment }\end{array}$ & $v_{a}$ & $\begin{array}{l}\text { number of time slots that appointment } a \text { is } \\
\text { scheduled after week } Z_{a}\end{array}$ \\
\hline$T$ & number of time slots in planning horizon & $p$ & difference between the number of appointment \\
\hline$\Theta$ & number of weeks delay in treatment process & & days realized and $\Omega$ \\
\hline$\Psi$ & prescribed duration of series of appointments & $\mu$ & excess number of non-recurring starting time slots \\
\hline$\Omega$ & minimal number of appointment days needed & & \\
\hline$U$ & $\begin{array}{l}\text { maximum allowed waiting time for the patient } \\
\text { between two consecutive appointments on a day }\end{array}$ & & \\
\hline
\end{tabular}

\section{Start of the rehabilitation process}

As we want to control the access time, we have to identify the number $f$ of the starting time slot of the very first appointment. Let parameter $F_{a c}$ be 1 if appointment $a$ is the first appointment for discipline $c$ according to the treatment plan and 0 otherwise. Then, we obtain the following:

$$
f=\min _{c}\left\{\sum_{a, h, t}\left(F_{a c} \cdot t \cdot x_{a h t}\right)\right\}
$$

Based on $f$, the number $k$ of the day on which the very first appointment takes place is as follows:

$k=\left\lceil\frac{1}{D} \cdot f\right\rceil$.

The access time of the patient should preferably be within $S$ weeks. Let $W$ be the number of time slots in a week and $N$ be 1 if the patient is a new patient and 0 otherwise. We introduce the variable $b$, which is the number of time slots by which the access time exceeds the preferred access time $(b \geq 0)$ :

$N \cdot(f-b) \leq S \cdot W \cdot$ 
We limit exceeding of the access time by requiring that $b$ may be no larger than $C$ times the preferred access time:

$b \leq C \cdot S \cdot W$.

Patients who cannot be seen within the preferred access time plus the maximum allowed extension, are instead referred to another rehabilitation clinic, as clinicians indicate that quality of care cannot be guaranteed when the access time exceeds this threshold.

For the rehabilitation process it is preferable that the patient starts treatment with all of the various relevant disciplines simultaneously. Therefore, we would like the first appointment with each discipline to take place within $V$ days of the very first appointment. We introduce the variable $m$, which is 1 if this preference is not satisfied, and penalize $m$ in the objective function:

$N \cdot \sum_{a, h, t}\left(F_{a c} \cdot t \cdot x_{a h t}\right) \leq D \cdot(k+V-1)+W \cdot m, \quad \forall c$.

\section{Continuity of the rehabilitation process}

For each appointment $a$, the treatment plan prescribes the range of weeks within which it should be scheduled (counting from the week in which the rehabilitation process started). Let $Y_{a}$ be the number of the first week in which $a$ may scheduled and $Z_{a}$ be the number of the final week. Now we would like to schedule $a$ in one of the weeks $Y_{a}, \ldots, Z_{a}$. As a deviation from these preferred weeks is better than not scheduling $a$ at all, we allow for some (penalized) scheduling flexibility: $a$ may be scheduled a week earlier than week $Y_{a}$ or a week later than week $Z_{a}$ if the patient does not already have an appointment with that same discipline during these other weeks. Hence, we first determine whether or not this situation applies. We introduce variables $q_{a}\left(r_{a}\right)$, which are 1 if appointment a may not be scheduled a week earlier (later) and 0 otherwise. Let $\mathcal{D}_{Y_{a}}$ denote the set of days in the week before week $Y_{a}$. Thus, we require the following:

$\sum_{h, c, t \in \mathcal{T}_{d}} \sum_{d \in \mathcal{D}_{Y_{a}}}\left(A_{h t}+\sum_{\hat{a}} x_{\hat{a} h t}\right) \cdot I_{a c} \cdot J_{h c} \leq L \cdot q_{a}, \quad \forall a$.

Similarly, if $\mathcal{D}_{Z_{a}}$ denotes the set of days in the week after week $Z_{a}$, we need the following:

$$
\sum_{h, c, t \in \mathcal{T}_{d}} \sum_{d \in \mathcal{D}_{Z_{a}}}\left(A_{h t}+\sum_{\hat{a}} x_{\hat{a} h t}\right) \cdot I_{a c} \cdot J_{h c} \leq L \cdot r_{a}, \quad \forall a .
$$

In case appointment $a$ has to be scheduled before week $Y_{a}$, the variable $u_{a}$ counts the number of time slots between the start of $a$ and the start of week $Y_{a}$. Now, $u_{a}$ may be at most a week, unless $a$ may not be scheduled earlier:

$u_{a} \leq W-W \cdot q_{a}, \quad \forall a$.

In case appointment $a$ has to be scheduled after week $Z_{a}$, the variable $v_{a}$ counts the number of time slots between the end of week $Z_{a}$ and the start of $a$, and we require the following:

$v_{a} \leq W-W \cdot r_{a}, \quad \forall a$.

Now, we would like to schedule each appointment $a$ in the week or range of weeks prescribed in the treatment plan or set $u_{a}\left(v_{a}\right)$ to the right value if $a$ is scheduled earlier (later) than prescribed. In the latter case, we penalize for this in the objective function. If $a$ can neither be scheduled in the prescribed weeks nor earlier or later, $a$ is not scheduled at all, and $n_{a}$ is set to 1 . Let $T$ be the total number of time slots in the planning horizon, $\Phi$ the number of days that have passed since the start of the rehabilitation process, and $\Theta$ the number of week-long delays since the start of the rehabilitation process. To not schedule $a$ too early, we require the following:

$$
\begin{array}{cl}
1+N \cdot D \cdot(k-1)+W \cdot\left(\Theta+Y_{a}-1\right)-u_{a} \leq & \forall a . \\
D \cdot \Phi+\sum_{h, t} t \cdot x_{a h t}+T \cdot n_{a}, &
\end{array}
$$

Similarly, to not schedule $a$ too late, we require the following:

$$
\begin{aligned}
& D \cdot \Phi+\sum_{h, t} t \cdot x_{a h t} \leq \\
& N \cdot D \cdot(k-1)+W \cdot\left(\Theta+Z_{a}\right)+v_{a}, \quad \forall a .
\end{aligned}
$$

The lead time of the rehabilitation process, from the first until the last appointment, should preferably be as prescribed in the treatment plan. It is undesirable to lengthen the lead time for scheduling reasons. Let $\Psi$ be the prescribed duration in weeks of a series of appointments. We introduce the variables $z_{1}, z_{2}$, and $z_{3}$. If the prescribed duration is exceeded by one week or less, $z_{1}$ is 1 . Otherwise, if the actual duration exceeds the prescribed duration by between one and two weeks both $z_{1}$ and $z_{2}$ are 1 . If the duration exceeds the prescribed length by more than two weeks, see the following $z_{3}$ is 1 :

$\max _{a, h, t}\left\{t \cdot x_{a h t}\right\}-N \cdot f \leq W \cdot\left(\Psi+z_{1}+z_{2}+T \cdot z_{3}\right)$.

When the prescribed duration is exceeded, we penalize this situation with the weights $\gamma_{1}, \gamma_{2}$, and $\gamma_{3}$ (for $z_{1}, z_{2}$, and $z_{3}$, respectively), where $\gamma_{1}<\gamma_{2}$ and $\gamma_{1}+\gamma_{2}<\gamma_{3}$.

\section{Patient preferences}

Combination appointments are high on the list of outpatient preferences [22]. Therefore, we strive to schedule the appointments on as few days as possible. We introduce a parameter $\Omega$ representing the minimal number of 'appointment days' required given the constraints of no more than $K$ appointments on a single day (11) and the fact that multiple appointments with the same therapist may not be scheduled for the same day (8). The variable $p$ that is penalized in the objective function represents the difference between the true number of 'appointment days' and $\Omega(p \geq 0)$ :

$$
\sum_{d} e_{d} \leq \Omega+p
$$

To limit patients' waiting time between appointments on the same day, these time intervals between two consecutive appointments in one day should not exceed $U$ time slots. Let $E_{t \hat{t}}$ be 1 if time slots $t$ and $\hat{t}$ fall on the same day. Thus, we have to require the following:

$$
\begin{gathered}
\sum_{\hat{t}=t+M_{a}+U+1}^{t+D} E_{t \hat{t}} \cdot \sum_{\hat{h}}\left(A_{\hat{h} \hat{t}}+\sum_{\hat{a}} x_{\hat{a} \hat{h} \hat{t}}\right) \leq \\
K \cdot \sum_{\hat{t}=t+M_{a}}^{t+M_{a}+U} E_{t \hat{t}} \cdot \sum_{\hat{h}}\left(A_{\hat{h} \hat{t}}+\sum_{\hat{a}} x_{\hat{a} \hat{h} \hat{t}}\right)
\end{gathered}
$$




\section{Recurring day and time}

It is preferred that the appointments take place on the same day and time each week, such that the patient has to remember only a short list of days and times. Hence, for an appointment that starts in time slot $t$, we first verify whether or not another appointment has been scheduled for the same time slot in one of the previous weeks (i.e., a multiple of $W$ time slots before $t$ ). If not, the binary variable $\tau_{t}$ is set to 1 , indicating that $t$ is a non-recurring appointment time slot:

$$
\begin{aligned}
x_{a h t}-\sum_{w \mid w \cdot W<t} \sum_{\hat{h}}\left(A_{\hat{h}(t-w \cdot W)}+\sum_{\hat{a}} x_{\hat{a} \hat{h}(t-w \cdot W)}\right) \leq \\
\tau_{t}, \quad \forall a, h, t .
\end{aligned}
$$

Clearly, the number of non-recurring appointment time slots is at least equal to the maximum number of appointments that take place within one week. We let the variable $\mu$ count and penalize the excess non-recurring appointment time slots by adding $\mu$ to the objective function. The constraint is as follows:

$\sum_{t} \tau_{t}-\mu \leq \max _{w}\left\{\sum_{t \in \mathcal{T}_{w}} \sum_{h}\left(A_{h t}+\sum_{a} x_{a h t}\right)\right\}$.

\section{Efficient filling of therapist schedules}

For the convenience of the therapists and to achieve a high utilization rate, it is preferable to avoid idle time in the schedules of therapists between two consecutive appointments in a day. As it might be impossible to later fit another appointment into this idle time, the prevention of idle time minimizes the number of referred patients and unscheduled appointments. Hence, we aim to schedule appointments right at the start or at the end of a session of the therapist, or right before or after an already scheduled appointment. We introduce the variable $i_{a}\left(j_{a}\right)$, which is 1 if appointment $a$ is scheduled in such a way that idle time is caused in the schedule of the therapist before (after) $a$. Then, we have to require the following:

$i_{a} \geq G_{h(t-1)} \cdot x_{a h t}, \quad \forall a, h, t$,

$j_{a} \geq G_{h\left(t+M_{a}\right)} \cdot x_{a h t}, \quad \forall a, h, t$.

If an appointment $a$ is scheduled in such a way that it causes idle time in the schedule of the therapist both beforehand and afterwards, we say that $a$ causes a break in the schedule of the therapist. This break is penalized in the objective function by the variable $g_{a}$, which is 1 in this case (and 0 otherwise):

$i_{a}+j_{a} \leq 1+g_{a}, \quad \forall a$.

\section{Objective function}

The objective function of the model, as presented in detail in Section 4.2.3, is as follows:

$$
\begin{aligned}
& \min \left\{\eta \cdot \sum_{a} n_{a}+\kappa \cdot \sum_{a} s_{a}+\alpha \cdot\left\lceil\frac{b}{D}\right\rceil+\beta \cdot m+\right. \\
& \left.\theta \cdot \sum_{a}\left\lceil\frac{u_{a}+v_{a}}{D}\right\rceil+\sum_{i=1}^{3} \gamma_{i} \cdot z_{i}+\delta \cdot p+\chi \cdot \mu+\zeta \cdot \sum_{a} g_{a}\right\}
\end{aligned}
$$

\section{References}

1. World Health Organization, The World Bank (2011) World report on disability. Available at: http://www.who.int/disabilities/world_report/2011/en/index. html

2. Wade DT, De Jong BA (2000) Recent advances in rehabilitation. British Medical Journal 320(7246):1385-1388

3. Kroll T, Neri M (2003) Experiences with care coordination among people with cerebral palsy, multiple sclerosis, or spinal cord injury. Disability and Rehabilitation 25(19):1106-1114

4. Conforti D, Guerriero F, Guido R (2008) Optimization models for radiotherapy patient scheduling. 4OR: A Quarterly Journal of Operations Research 6(3):263-278

5. Dafoe W, Arthur H, Stokes H, Morrin L, Beaton L, et al. (2006) Universal access: but when? Treating the right patient at the right time: access to cardiac rehabilitation. Canadian Journal of Cardiology 22(11):905-911

6. Scheer J, Kroll T, Neri MT, Beatty P (2003) Access barriers for persons with disabilities. Journal of Disability Policy Studies 13(4):221-230

7. Kok L, Houkes A, Niessen N (2008) Kosten en baten van revalidatie [Costs and benefits of rehabilitation]. SEO-report 2008-68, SEO Economisch Onderzoek, Amsterdam. Available at: http://www.revalidatienederland.nl/actueel/publicaties (in Dutch)

8. De Jong, M (2010) Revalidatie in Beweging [Rehabilitation on the move]. CC zorgadviseurs, Woerden. Available at: http://www.revalidatienederland.nl/actueel/publicaties (in Dutch)

9. De Bleser L, Depreitere R, De Waele K, Vanhaecht K, Vlayen J, Sermeus W (2006) Defining pathways. Journal of Nursing Management 14(7):553-563

10. Pierskalla WP, Brailer JD (1994) Applications of operations research in health care delivery. Handbooks in OR \& MS, 6:469-505

11. Brandeau ML, Sainfort F, Pierskalla WP, editors (2004) Operations Research and Health Care: a Handbook of Methods and Applications. International Series in Operations Research and Managmenent Science, Vol. 70, Kluwer Academic Publishers, Boston, MA

12. Lagergren $M$ (1998) What is the role and contribution of models to management and research in the health services? A view from Europe. European Journal of Operational Research 105(2):257-266

13. Cayirli T, Veral E (2003) Outpatient scheduling in health care: a review of literature. Production and Operations Management 12(4):519-549

14. Gupta D, Denton B (2008) Appointment scheduling in health care: Challenges and opportunities. IIE Transactions 40(9):800-819

15. Chien CF, Tseng FP, Chen CH (2008) An evolutionary approach to rehabilitation patient scheduling: A case study. European Journal of Operational Research 189(3):12341253

16. Chien CF, Huang YC, Hu CH (2009) A hybrid approach of data mining and genetic algorithms for rehabilitation scheduling. International Journal of Manufacturing Technology and Management 16(1):76-100

17. Podgorelec V, Kokol P (1997) Genetic algorithm based system for patient scheduling in highly constrained situations. Journal of Medical Systems 21(6):417-427

18. Ogulata SN, Koyuncu M, Karakas E (2008) Personnel and patient scheduling in the high demanded hospital services: a case study in the physiotherapy service. Journal of Medical Systems 32(3):221-228 
19. Conforti D, Guerriero F, Guido R, Veltri M (2011) An optimal decision-making approach for the management of radiotherapy patients. OR Spectrum 33(1):123-148

20. Turkcan A, Zeng B, Lawley M (2012) Chemotherapy operations planning and scheduling. IIE Transactions on Healthcare Systems Engineering 2(1):31-49

21. Beattie P, Dowda M, Turner C, Michener L, Nelson R (2005) Longitudinal continuity of care is associated with high patient satisfaction with physical therapy. Physical Therapy 85(10):1046-1052

22. Vermeulen I, Bohte S, Elkhuizen S, Bakker P, La Poutré H (2008) Decentralized online scheduling of combinationappointments in hospitals. In Proceedings of ICAPS-2008, the International Conference on Automated Planning and Scheduling, Sydney, Australia, pages 372-379

23. Law AM, Kelton WD (2000) Simulation modeling and analysis. McGraw-Hill, New York, NY 